\title{
Upregulation of Myc promotes the evasion of NK cell-mediated immunity through suppression of NKG2D ligands in K562 cells
}

\author{
YOUNG-SHIN LEE ${ }^{1,2^{*}}$, WOONG HEO ${ }^{1,2}$, CHEOL-HUN SON $^{3 *}$, CHI-DUG KANG $^{1}$, \\ YOU-SOO PARK ${ }^{3}$ and JAEHO BAE ${ }^{1,2}$ \\ ${ }^{1}$ Department of Biochemistry, Pusan National University School of Medicine; ${ }^{2}$ Department of Molecular Cell Biology \\ and Genetics, PNU BK21 Plus Biomedical Science Education Center, Pusan National University School of Medicine, \\ Yangsan, Gyeongsangnam 50612; ${ }^{3}$ Department of Research Center, Dongnam Institute of Radiological and \\ Medical Sciences, Gijang, Busan 46033, Republic of Korea
}

Received November 12, 2018; Accepted August 7, 2019

DOI: $10.3892 / \mathrm{mmr} .2019 .10583$

\begin{abstract}
Myc is a characteristic oncogene with dual functions in cell proliferation and apoptosis. Since the overexpression of the c-Myc proto-oncogene is a common event in the development and growth of various human types of cancer, the present study investigated whether oncogenic c-Myc can alter natural killer (NK) cell-mediated immunity through the expression of associated genes, using PCR, western blotting and flow cytometry assays. Furthermore, whether c-Myc could influence the expression levels of natural killer group 2 member D (NKG2D) ligands, which are well known NK activation molecules, as well as NK cell-mediated immunity, was investigated. c-Myc was inhibited by 10058-F4 treatment and small interfering RNA transfection. Upregulation of c-Myc was achieved by transfection with a pCMV6-myc vector. The inhibition of c-Myc increased MHC class I polyeptide-related sequence B and UL16 binding protein 1 expressions among NKG2D ligands, and the overexpression of c-Myc suppressed the expression of all NKG2D ligands, except MHC class I polyeptide-related sequence A. Furthermore, the alteration of c-Myc activity altered the susceptibility of K562 cells to NK cells. These results suggested that the overexpression of c-Myc may contribute to the immune escape of cancer cells
\end{abstract}

Correspondence to: Professor Jaeho Bae, Department of Biochemistry, Pusan National University School of Medicine, 49 Busandaehak-ro, Yangsan, Gyeongsangnam 50612, Republic of Korea

E-mail: biosole@pusan.ac.kr

Dr You-Soo Park, Department of Research Center, Dongnam Institute of Radiological and Medical Sciences, 40 Jwadong-gil, Gijang, Busan 46033, Republic of Korea

E-mail: biotek01@hanmail.net

*Contributed equally

Key words: c-Myc, natural killer group 2 member D ligands, 10058-F4, K562 and cell proliferation. Combined treatment with NK-based cancer immunotherapy and inhibition of c-Myc may achieve improved therapeutic results.

\section{Introduction}

Most of the oncogenes have been formed by mutation, amplification, and rearrangement of proto-oncogenes, which regulate cell growth, cell differentiation, and apoptosis. Overexpression or hyperactivation of these oncogenes drives uncontrolled cell proliferation and resistance to apoptosis, which are the main characteristics of cancer cells (1). c-Myc is one of the key oncogenes responsible for many human cancers, and unlike normal cells, it is aberrantly expressed in cancer cells (2-4). In addition, c-Myc also induces many genes such as eIF-2, eIF-4E, p53, cyclin D/E, CDK4, CDC25A, and p19/p14ARF, and facilitates degradation of p27 $(5,6)$. However, it is not known clearly whether c-Myc is related to the expression of natural killer group 2 member D (NKG2D) ligands and the susceptibility of cancer cells to NK cells. Since NK cells and $\gamma \delta-\mathrm{T}$ cells recognize cancer cells through NKG2D ligands and eliminate them (7), induction of NKG2D ligands in cancer cells is very critical to evoke NK cell-mediated anti-cancer immunity. It was well known that normal cells, except activated T-cells and some tissue cells, such as in the intestinal epithelium, generally do not express NKG2D ligands $(8,9)$; besides, the abundant expression of MHC class molecules on their surface also protect the normal cells from the activated NK cells (10). However, the potency of immune surveillance in patients is not sufficient to eliminate transformed cells through the process called 'lack of induction', which means insufficient induction of NK activating ligands $(11,12)$. Since aberrant expression of c-Myc oncogenes changes the expression of many other genes, and maintains malignancy in cancer cells, it was suspected that c-Myc might disturb NK cell-mediated immune responses through the altered expression of several genes, such as those encoding NKG2D ligands. Previously, it was revealed that inhibition of several oncogenes, such as EGFR, PI3K, Ras, NF- $\kappa \mathrm{B}$ and BCR/ABL, could modulate the expression of NKG2D ligands (13-16). In this study, we investigated whether c-Myc modulates the expression of 
NKG2D ligands and affects the susceptibility of cancer cells to NK cells.

\section{Materials and methods}

Cell lines and reagents. The K562 chronic myeloid leukemia cell line was available with the Korean Cell Line Bank (Seoul, Korea) originated from ATCC (@CCL-243). These cells were maintained in RPMI medium (Invitrogen; Thermo Fisher Scientific, Inc.) supplemented with $10 \%$ fetal bovine serum (FBS) (Gibco; Thermo Fisher Scientific, Inc.), 2 mM L-glutamine, $100 \mu \mathrm{g} / \mathrm{ml}$ streptomycin, and $100 \mathrm{U} / \mathrm{ml}$ penicillin. The NK-92 cell line was obtained from the American Type Culture Collection and maintained in alpha-Minimum Essential Modified medium supplemented with $12.5 \%(\mathrm{v} / \mathrm{v})$ FBS, $12.5 \%(\mathrm{v} / \mathrm{v})$ horse serum, $2 \mathrm{mM}$ L-glutamine, $0.1 \mathrm{mM}$ 2-mercaptoethanol, $200 \mathrm{U} / \mathrm{ml}$ of recombinant human interleukin-2, $100 \mu \mathrm{g} / \mathrm{ml}$ streptomycin, and $100 \mathrm{U} / \mathrm{ml}$ penicillin. All cell lines were maintained at $37^{\circ} \mathrm{C}$ in a humidified atmosphere containing 5\% $\mathrm{CO}_{2}$. c-Myc inhibitor, 10058-F4, which prevented the binding of c-Myc/Max dimers to its DNA targets, was purchased from Calbiochem.

Total RNA extraction and multiplex reverse transcription $(R T)-P C R$. Total cellular RNA was extracted using RNeasy ${ }^{\circledR}$ Mini Kit (Qiagen $\mathrm{GmbH}$ ) by following the manufacturer's protocol. cDNA was synthesized from $1 \mu \mathrm{g}$ of extracted total RNA using $100 \mathrm{pmol}$ of random primers (Takara) and 100 units of M-MLV reverse transcriptase (Promega Corporation). The synthesized cDNA was used in the PCR reaction with reagents in the QIAGEN ${ }^{\circledR}$ Multiplex PCR kit (Qiagen GmbH). Seven pairs of primer sets were used to investigate the expression of genes, including ribosomal protein L19 (RPL19), MICA, MICB, ULBP1-3 and $\beta$-actin (ACTB) (17). ACTB and RPL19 were used as a loading control and degradation marker, respectively. The PCR products were analyzed using ethidium bromide-stained $2.0 \%$ agarose gel electrophoresis and quantitated by image analyzing software, Quantity One (Bio-Rad Laboratories, Inc.).

c-Myc silencing using siRNA transfection. K562 cells were transfected with c-Myc targeting small interfering RNA (siRNA) or scrambled RNA (scRNA) using Oligofectamine ${ }^{\mathrm{TM}}$ Reagent (Life Technologies; Thermo Fisher Scientific, Inc.) by following the manufacturer's protocol. Chemically synthesized siRNA and scRNA were purchased from Bioneer. The cells were treated with $200 \mathrm{nM}$ final concentration of siRNA/scRNA and harvested after incubation for 24,48 and $72 \mathrm{~h}$.

c-Myc overexpression in K562 cells using pCMV6 vector. pCMV6 and pCMV6-myc vectors were purchased from Origene. Each vector was transfected into K562 cells using $\mathrm{Xfect}^{\mathrm{TM}}$ Transfection Reagent (Clontech) by following the manufacturer's protocol. After $24 \mathrm{~h}$ of incubation, cells were distributed into 12 -well and selected by treatment with $0.8 \mathrm{mg} / \mathrm{ml}$ of G418 (Geneticin ${ }^{\circledR}$; Life Technologies; Thermo Fisher Scientific, Inc.). Positive cells were maintained in RPMI medium containing $100 \mu \mathrm{g} / \mathrm{ml}$ of G418.

Western blot analysis. Cells were washed with ice-cold phosphate buffer, lysed in lysis buffer consisting of $1 \%(\mathrm{w} / \mathrm{v})$ sodium dodecyl sulfate (SDS), $1.0 \mathrm{mM}$ sodium ortho-vanadate,

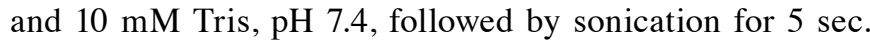
Proteins in the cell lysate were quantified using a Bradford protein assay kit (Pierce). Proteins were separated by $10 \%$ SDS-polyacrylamide gel electrophoresis (SDS-PAGE) using a mini gel apparatus (Bio-Rad Laboratories, Inc.) and were transferred onto nitrocellulose membranes (Hybond-ECL; GE Healthcare). Each membrane was blocked with 5\% skimmed milk in Tris-buffered saline containing $0.05 \%$ Tween-20 (TBST). Protein bands were probed with primary antibody, followed by labeling with horseradish peroxidaseconjugated anti-mouse, anti-rabbit secondary antibody (Cell Signaling Technology). The primary antibodies used were: c-Myc (Epitomics), $\beta$-actin antibody (Sigma-Aldrich; Merck KGaA). Bands were visualized by enhanced chemiluminescence (Amersham Pharmacia Biotech) according to manufacturer's instruction and the densities were measured by Multi Gauge v3.0 (Fujifilm Medical Systems Inc.).

Flow cytometry analysis of NKG2D ligands. To determine the surface expression of the NKG2D ligands on cancer cells, the cells were incubated with mouse anti-MICA, anti-MICB, and anti-ULBP1-3 (R\&D Systems), which were NKG2D ligand-specific monoclonal antibodies (mAbs), and the corresponding isotype controls at $10 \mu \mathrm{g} / \mathrm{ml}$, followed by incubation with the goat anti-mouse-PE conjugated (BD Pharmingen Inc.). The analysis was performed on the FACSCalibur ${ }^{\circledR}$ system using the CellQuest software (both from Becton-Dickinson), and the cell surface expression was quantified from the value of the mean fluorescence intensities (MFI) obtained with the specific mAbs.

NK cell-mediated cytotoxicity assay. NK cell-mediated cytotoxicity was determined using flow cytometry. Briefly, untreated, 10058-F4 treated, and c-Myc upregulated K562 cells were harvested. The cells were stained with $50 \mu \mathrm{M}$ CFSE for $30 \mathrm{~min}$ at $37^{\circ} \mathrm{C}$ and washed three times. NK-92 cells and CFSE-stained K562 cells were co-cultured for $4 \mathrm{~h}$. Propidium iodide (PI) was added to the co-cultured samples to mark dead cells. The proportion of dead cells was analyzed by formula: $\left(\mathrm{CFSE}^{+} \mathrm{PI}^{+}\right.$cells/CFSE ${ }^{+}$cells) $\mathrm{x} 100$.

Statistical analysis. To evaluate the altered level of gene expression, the mean folds of gene expression were calculated. For comparison of groups, one way ANOVA was performed using SPSS software (version 11.01; SPSS, Inc.). P $<0.05$ was considered to indicate a statistically significant difference.

\section{Results}

c-Myc inhibitor increases the transcription of MICB and $U L B P 1$. Using multiplex-PCR reaction, the expression of 7 genes, including those for 5 NKG2D ligands and 2 housekeeping genes were analyzed, and were normalized to the level of ACTB. K562 cells were treated with 10058-F4, which selectively prevents the c-Myc-Max complex from interaction (18). MICA was further transcribed by treatment with 7.5 and $30 \mu \mathrm{M}$ of 10058-F4. The levels of MICB and ULBP1 transcripts were increased dose-dependently. The change in the level of transcription of ULBP2 and ULBP3 was not significant after treatment with 10058-F4 (Fig. 1). It was 
A

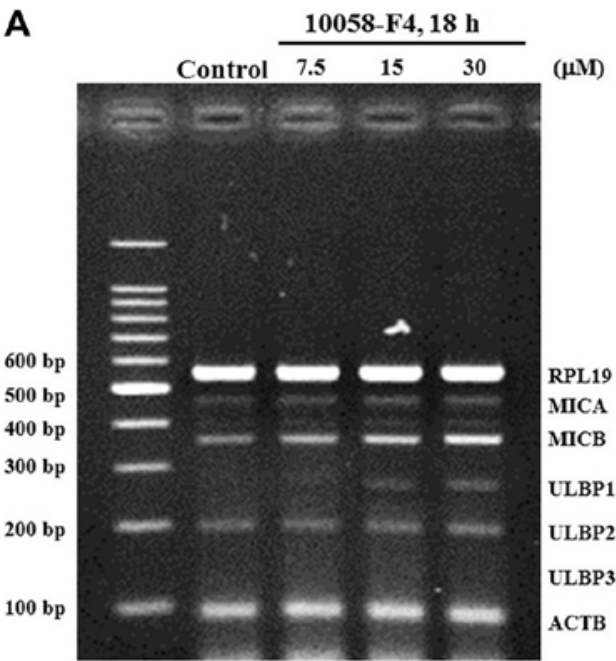

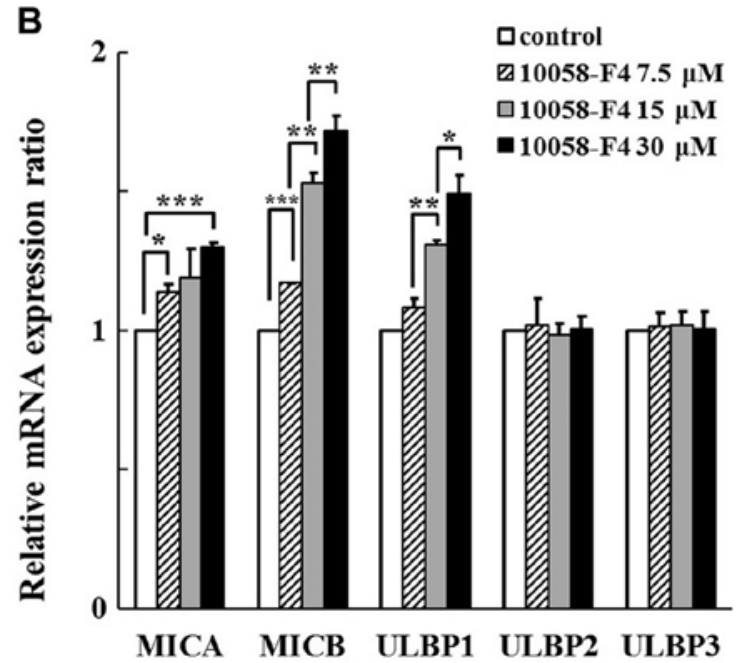

Figure 1. Analysis of NKG2D ligand transcription following treatment of K562 cells with 10058-F4. K562 cells were treated with 10058-F4, a c-Myc inhibitor, at indicated doses, for $18 \mathrm{~h}$. Subsequently, NKG2D ligand mRNA expression was analyzed using (A) multiplex reverse transcription-PCR and (B) relative expression ratios were compared with those of untreated control using image analyzing software (Quantity One). ${ }^{*} \mathrm{P}<0.05,{ }^{* *} \mathrm{P}<0.01$ and ${ }^{* * * *} \mathrm{P}<0.001$. ACTB, actin- $\beta$; MIC, MHC class I polypeptide-related sequence; NKG2D, natural killer group 2 member D; RPL19, ribosomal protein L19; ULBP, UL16 binding protein.
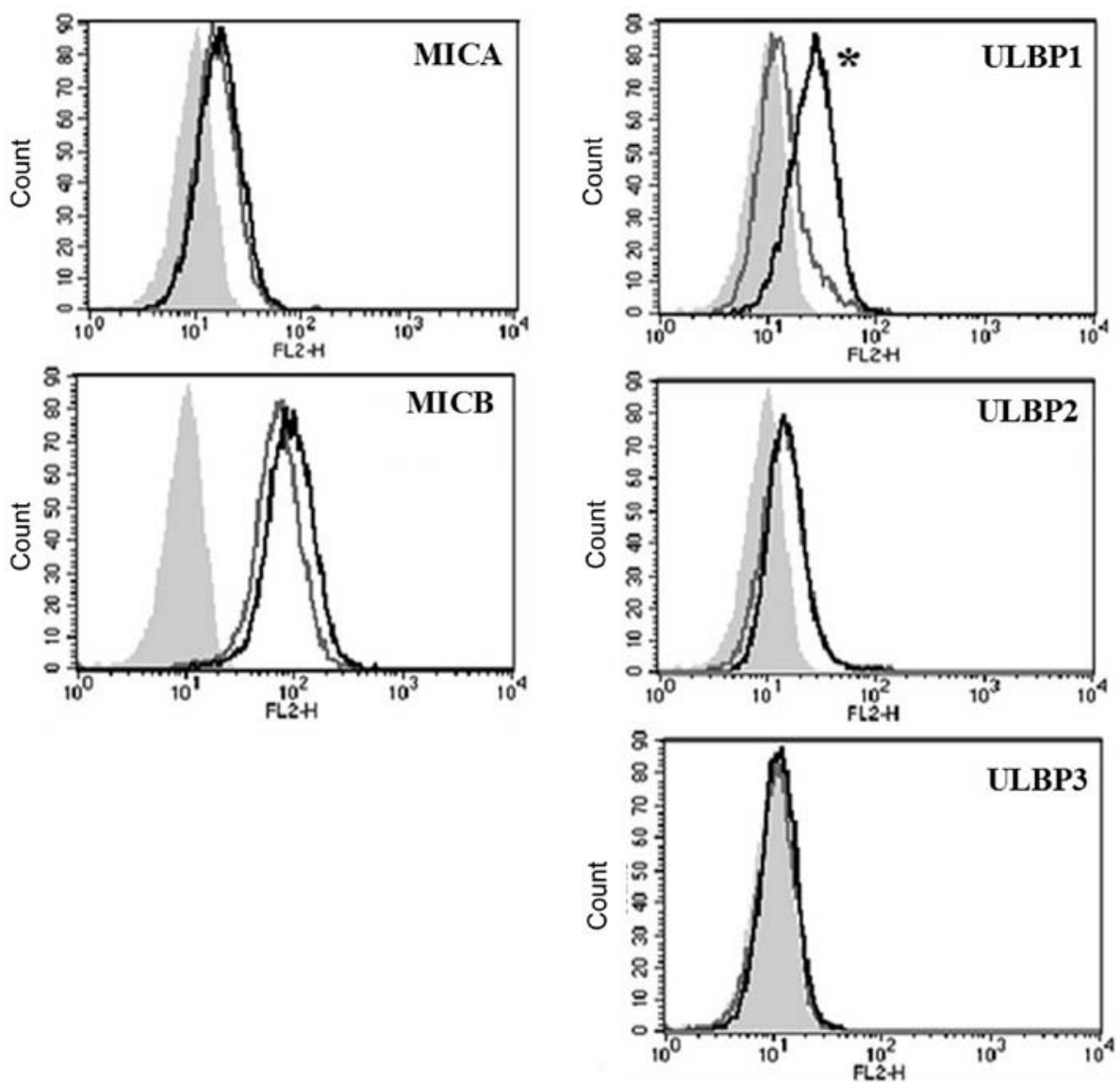

Figure 2. Natural killer group 2 member D ligand expression following treatment of K562 cells with 10058-F4. After 10058 -F4 treatment for 24 h at $15 \mu \mathrm{M}$, the expression of surface proteins was analyzed using flow cytometry. Filled gray, gray and black lines represent isotype, untreated control and 10058-F4 treatment, respectively. ${ }^{~} \mathrm{P}<0.05$. MIC, MHC class I polypeptide-related sequence; ULBP, UL16 binding protein.

supposed that c-Myc would significantly affect the expression of MICA, MICB, and ULBP1.

10058-F4 increases the surface expression of MICB and $U L B P 1$. To observe whether the inhibition of c-Myc could induce the expression of surface proteins of NKG2D ligands, the surface proteins were investigated using flow cytometry with specific antibodies after treatment with 10058-F4 and c-Myc inhibitor for $24 \mathrm{~h}$. Histograms of 5 NKG2D ligands were presented (Fig. 2). The expression of ULBP1 was 
prominently increased, whereas the expression of MICB was slightly increased. The expression of MICA and ULBP2-3 on the surface were not altered by treatment with 10058-F4.

Silencing of $c-M y c$ using siRNA induces the expression of $M I C B, U L B P 1$ and ULBP3 mRNA. c-Myc gene was successfully silenced by transfection with c-Myc siRNA in a time dependent manner (Fig. 3A). c-Myc Silencing induced the expression of MICB, ULBP1, and ULBP3 at the transcriptional level (Fig. 3B). ULBP1 expression was significantly induced after $48 \mathrm{~h}$ in a time-dependent manner. From these c-Myc inhibition tests using 10058-F4 and siRNA, it could be indicated that c-Myc might suppress the expression of MICB and ULBP1.

MICB expression is suppressed through the upregulation of $c-M y c$ and restored by $c$-Myc inhibition. To study whether the upregulation of c-Myc could suppress the expression of NKG2D ligands, pCMV or pCMV6-Myc vector was transfected into K562 cells and the transfected cells were isolated by selection using G418. In c-Myc upregulated K562 cells, surface proteins of all five NKG2D ligands have a trends of reduction compared with that in pCMV transfected K562 cells. Especially, MICB and ULBP 2 were decreased with statistically significance (Fig. 4A). Although the precise mechanisms are not clear, it was obvious that c-Myc could inhibit the expression of NKG2D ligands. When c-Myc inhibitor was added to the c-Myc upregulated cells, the expression of the suppressed NKG2D ligands such as MICB and ULBP1/2 was restored (Fig. 4B). Therefore, it could be suggested that c-Myc negatively affected the expression of NKG2D ligands.

Upregulation of c-Myc suppresses the susceptibility of K562 cells to NK-92 cells. To confirm whether c-Myc could affect the susceptibility of K562 cells to NK-92 cells, K562 cells were co-cultured with NK-92 cells and the proportion of dead cells was determined through PI staining. The inhibition of c-Myc increased the susceptibility of K562 cells to NK-92 cells (Fig. 5A) and the upregulation of c-Myc decreased the susceptibility (Fig. 5). Therefore, it could be concluded that c-Myc suppressed the susceptibility of K562 cells to NK-92, possibly through the down-regulation of NKG2D ligand expression.

\section{Discussion}

c-Myc belongs to the MYC family, most members of which are located on chromosome 8 and is related to cell proliferation, cell growth, and apoptosis. It was reported that malfunctioning of c-Myc leads to cancers of the breast, cervix, colon, stomach, lungs, and leukocytes (2-4). Since c-Myc influences the expression of many genes $(5,6)$, c-Myc overexpression in cancer cells is a factor behind poor prognosis (19). It was suspected that c-Myc might attenuate body defense against cancer and disturb immune system through the altered expression of NKG2D ligands, which are a kind of NK-cell activating ligands. In this study, we investigated whether c-Myc affected the expression of NKG2D ligands in chronic myeloid leukemia cells (K562 cells). The K562 cells express BCR/ABL, a fusion oncoprotein, as well as abundant c-Myc $(20,21)$. Since K562 cells show impairment in the functions of $\mathrm{p} 53$, which mediates
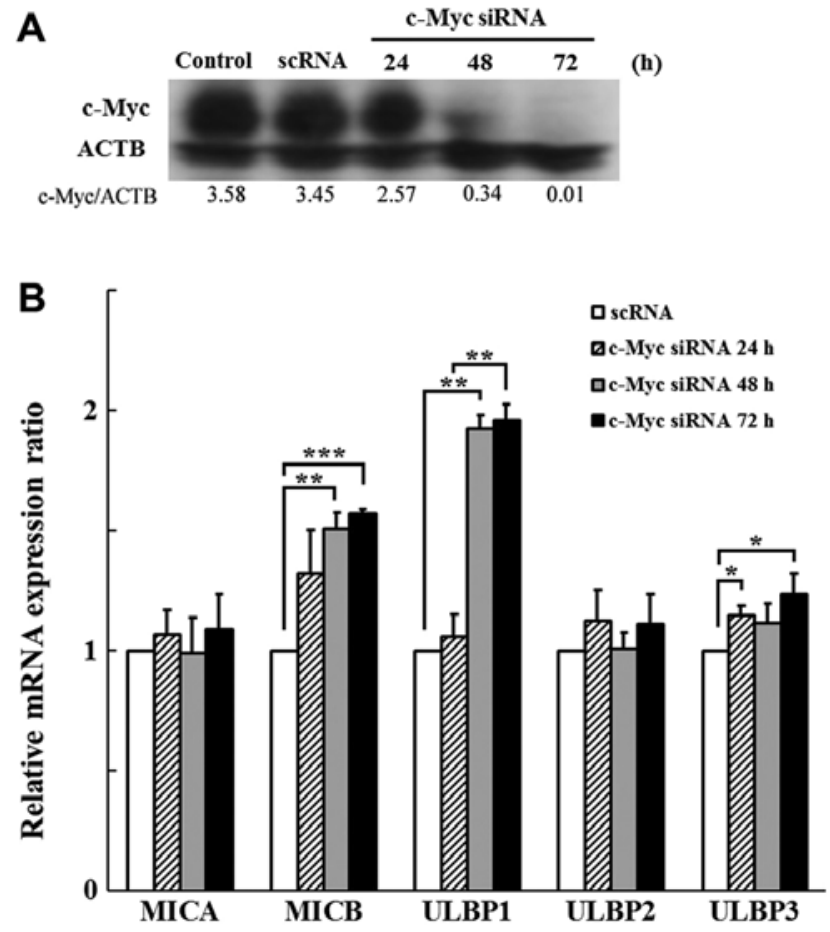

Figure 3. Analysis of NKG2D ligand expression following knockdown of c-Myc using siRNA in K562 cells. (A) K562 cells were transfected with scRNA and c-Myc siRNA. Efficiency of c-Myc siRNA in K562 cells was confirmed by western blotting. The densities were measured by Multi Gauge software and presented as the ratio to ACTB. (B) Expression levels of NKG2D ligands were analyzed using multiplex-PCR at 24,48 and $72 \mathrm{~h}$ post siRNA transfection. The altered expression levels were represented as ratios relative to scRNA transfected cells. ${ }^{*} \mathrm{P}<0.05,{ }^{* *} \mathrm{P}<0.01,{ }^{* * * *} \mathrm{P}<0.001$. ACTB, actin- $\beta$; c-Myc siRNA, c-Myc targeted siRNA; MIC, MHC class I polypeptide-related sequence; NKG2D, natural killer group 2 member D; scRNA, scrambled RNA; siRNA, small interfering RNA; ULBP, UL16 binding protein.

Myc-induced apoptosis $(22,23)$, and since c-Myc transduction did not enhance apoptosis of K562 cells (data not shown), it was thought that K562 cells are adjustable to test viability and susceptibility to NK cells in this study after alteration of c-Myc expression.

Although it was generally known that transformed cells overexpressed NKG2D ligands, established cancer cells acquired resistance against host immune systems and escaped immune surveillance $(24,25)$. Previously, it was demonstrated that NKG2D ligand is not always upregulated in cancer cells when compared with the adjacent normal cells (17). Therefore, we hypothesized that the expression of NKG2D ligands on established cancer cells was insufficient to evoke NK-cell-mediated anti-cancer immune responses. It is already known that cancer cells use several immune suppressive mechanisms to escape from NK cells, such as secretion of TGF-beta and interleukin-6, increased extracellular shedding of NKG2D ligands accompanying reduction in surface NKG2D ligands, and increase of anti-apoptotic molecule (26-28). Since successful NK-cell-based anti-cancer immunotherapy depends on overcoming these immune escape mechanisms of cancer cells, searching for molecules that affect NK-cell-mediated immune responses was considered necessary.

Except BCR/ABL, which induces the expression of NKG2D ligands (14), inhibition of several oncogenes including Ras, EGFR, NF- $\mathrm{kB}$, and Akt can induce the expression of 

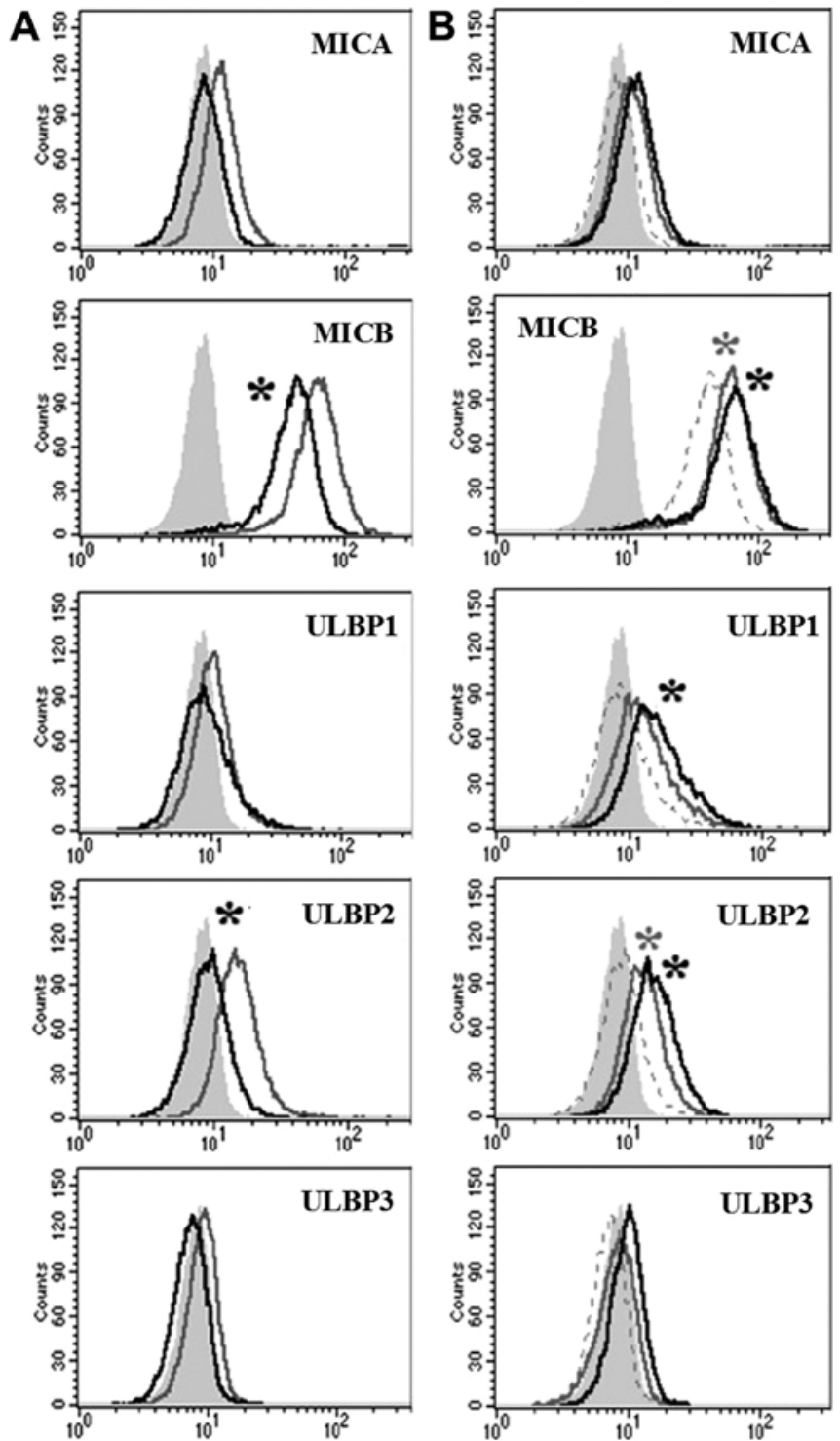

Figure 4. Analysis of the expression levels of NKG2D ligands in c-Myc-upregulated K562 cells. (A) Surface expression levels of NKG2D ligands were analyzed using flow cytometry at $48 \mathrm{~h}$ after pCMV-Myc vector transfection. Filled gray, gray and black lines represent isotype, pCMV and pCMV-Myc transfected cells, respectively. ${ }^{*} \mathrm{P}<0.05$ pCMV6-empty transfected cells vs. pCMV6-Myc transfected cells. (B) Once isolated, stable c-Myc upregulated K562 cells were treated with $15 \mu \mathrm{M}$ of $10058-\mathrm{F} 4$ for $24 \mathrm{~h}$ and the surface expression of NKG2D ligands was analyzed using flow cytometry. Filled gray, dot, gray and black lines represent isotype, pCMV6-Myc transfected cells, pCMV6-Myc transfected cells treated with $15 \mu \mathrm{M} 10058-\mathrm{F} 4$ and pCMV6-Myc transfected cells treated with $30 \mu \mathrm{M} 10058-\mathrm{F} 4$, respectively. "P<0.05 pCMV6-Myc transfected cells vs. pCMV6-Myc transfected cells treated with 15 (gray) or $30 \mu \mathrm{M}$ (black) 10058-F4. MIC, MHC class I polypeptide-related sequence; NKG2D, natural killer group 2 member D; ULBP, UL16 binding protein.

NKG2D ligands, therefore, it was thought that these oncogenes might contribute to immune escape of cancer cells $(13,15,16)$. However, it remains to be addressed whether c-Myc could modulate the expression of NKG2D ligands. In the present study, we found that overexpression of c-Myc decreased the expression of NKG2D ligands including MICA, MICB, and ULBPs, and the inhibition of c-Myc could restore the expression of NKG2D ligands. Depending on the level of NKG2D ligands, the activity of NK cells was altered.
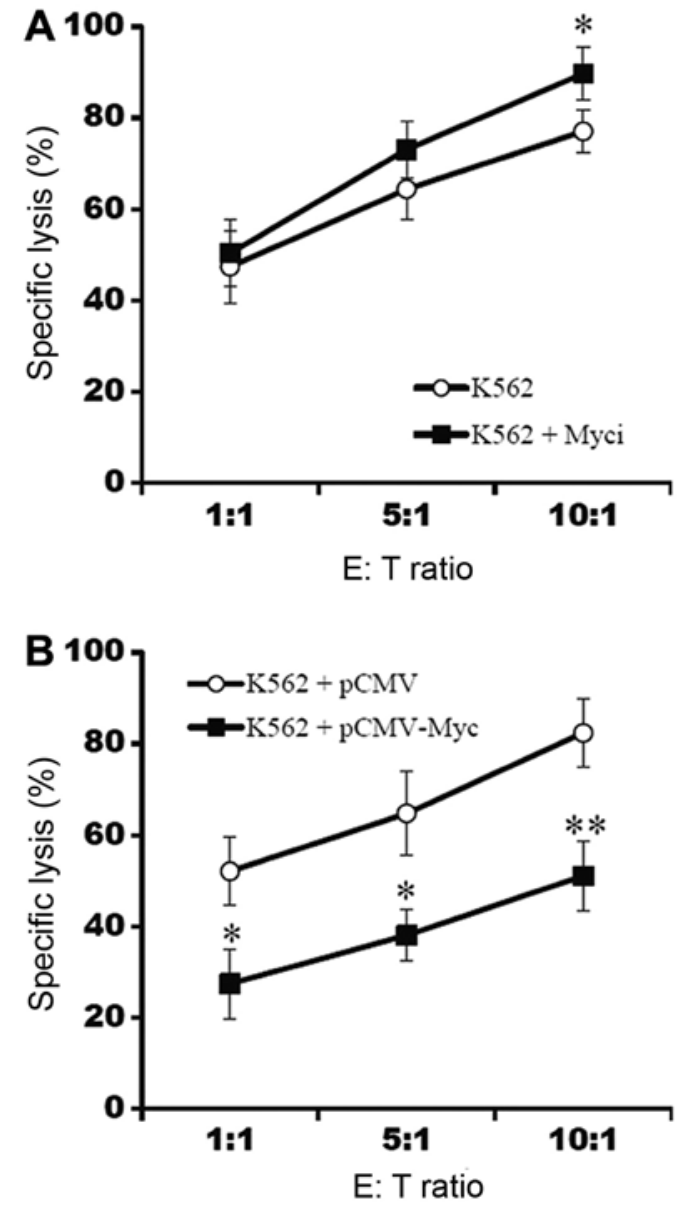

Figure 5. Analysis of the cytotoxicity of NK92-cells to K562 cells following inhibition or upregulation of c-Myc. Natural killer cell-mediated cytotoxicity was determined using flow cytometry after staining the target cells with carboxyfluorescein succinimidyl ester, and the dead cells with propidium iodide. (A) K562 cells were treated with c-Myc inhibitor (15 $\mu \mathrm{M} 10058-\mathrm{F} 4)$ for $24 \mathrm{~h}$, and co-cultured with NK-92 cells for $4 \mathrm{~h} .{ }^{*} \mathrm{P}<0.05$ vs. K562. (B) pCMV6 and pCMV6-Myc transfected K562 cells were co-cultured with NK-92 cells for 4 h. ${ }^{*} \mathrm{P}<0.05,{ }^{* *} \mathrm{P}<0.01$ vs. K562 + pCMV. E:T ratio, effector to target ratio. Myci, c-Myc inhibitor.

Gasser et al (29) demonstrated that tumorigenesis of ovarian epithelial cells by transduction with c-Myc did not induce the expression of NKG2D ligands. Although these authors did not assay inhibition of c-Myc in upregulated cells, they showed that the transplanted cells had increased level of NKG2D ligands (29). On the contrary, Nanbakhsh et al (30) showed that c-Myc had a role as a transcription factor in the expression of ULBP1/3 in cytarabine-resistant acute myeloid leukemia cells. Since cytarabine interferes with DNA synthesis and accumulates DNA damage, DNA repair systems, which are key regulators of NKG2D ligands, might complicate the results in the resistant cells. Although it was not quite clear why c-Myc differently affected the expression of NKG2D ligands in cytarabine-resistant acute myeloid leukemia cells and K562 chronic myeloid leukemia cells, a variety of functions of the hyperactivated c-Myc in tumorigenesis and secondary reactions in varied cancer types might lead to the differential expression of NKG2D ligands.

In conclusion, this study demonstrated that inhibition of c-Myc induces NKG2D ligands in K562 cells, and enhances their susceptibility to NK cells. Although there remain many 
unsolved questions, inhibition of c-Myc might contribute to better therapeutic outcome in the treatment of cancer patients when combined with NK-cell-based cancer immunotherapy in future.

\section{Acknowledgements}

Not applicable.

\section{Funding}

This work was supported by the Dongnam Institute of Radiological and Medical Sciences grant funded by the Korean government (MSIT; grant no. 50595-2018).

\section{Availability of data and materials}

The datasets used and/or analyzed during the current study are available from the corresponding author on reasonable request.

\section{Authors' contributions}

YSL performed experiments, including PCR and flow cytometry, and wrote the manuscript. WH performed experiments, including western blotting and cytotoxicity assays. CHS performed gene transfection. CDK developed the platform of multiplex PCR for NKG2D ligands. YSP performed statistical analysis and interpretation of data. JB designed and evaluated the study.

\section{Ethics approval and consent to participate}

Not applicable.

\section{Patient consent for publication}

Not applicable.

\section{Competing interests}

The authors declare that they have no competing interests.

\section{Authors' information}

YSL: MS researcher at Department of Biochemistry, Pusan National University School of Medicine; Department of Molecular Cell Biology and Genetics, PNU BK21 Plus Biomedical Science Education Center, Pusan National University School of Medicine, Yangsan, Gyeongsangnam 50612, Republic of Korea. WH: MS researcher at Department of Biochemistry, Pusan National University School of Medicine; Department of Molecular Cell Biology and Genetics, PNU BK21 Plus Biomedical Science Education Center, Pusan National University School of Medicine, Yangsan, Gyeongsangnam 50612, Republic of Korea. CHS: PhD. Senior researcher at Department of Research Center, Dongnam Institute of Radiological and Medical Sciences, Gijang, Busan 46033, Republic of Korea. CDK: Professor at Department of Biochemistry, Pusan National University School of Medicine,
Yangsan, Gyeongsangnam 50612, Republic of Korea. YSP: $\mathrm{PhD}$ director at Department of Research Center, Dongnam Institute of Radiological and Medical Sciences, Gijang, Busan 46033, Republic of Korea. JB: Associate Professor at Department of Biochemistry, Pusan National University School of Medicine; Department of Molecular Cell Biology and Genetics, PNU BK21 Plus Biomedical Science Education Center, Pusan National University School of Medicine, Yangsan, Gyeongsangnam 50612, Republic of Korea.

\section{References}

1. Shortt $\mathrm{J}$ and Johnstone RW: Oncogenes in cell survival and cell death. Cold Spring Harb Perspect Biol 4: pii: a009829, 2012.

2. Dang CV: MYC on the path to cancer. Cell 149: 22-35, 2012.

3. Vennstrom B, Sheiness D, Zabielski J and Bishop JM: Isolation and characterization of c-myc, a cellular homolog of the oncogene (v-myc) of avian myelocytomatosis virus strain 29. J Virol 42: 773-779, 1982.

4. Zech L, Haglund U, Nilsson K and Klein G: Characteristic chromosomal abnormalities in biopsies and lymphoid-cell lines from patients with Burkitt and non-Burkitt lymphomas. Int J Cancer 17: 47-56, 1976.

5. Dang CV: c-Myc target genes involved in cell growth, apoptosis, and metabolism. Mol Cell Biol 19: 1-11, 1999.

6. Grandori $\mathrm{C}$ and Eisenman RN: Myc target genes. Trends Biochem Sci 22: 177-181, 1997.

7. Bauer S, Groh V, Wu J, Steinle A, Phillips JH, Lanier LL and Spies T: Activation of NK cells and T cells by NKG2D, a receptor for stress-inducible MICA. Science 285: 727-729, 1999.

8. Diefenbach A, Jamieson AM, Liu SD, Shastri N and Raulet DH: Ligands for the murine NKG2D receptor: Expression by tumor cells and activation of NK cells and macrophages. Nat Immunol 1: 119-216, 2000.

9. Groh V, Bahram S, Bauer S, Herman A, Beauchamp M and Spies T: Cell stress-regulated human major histocompatibility complex class I gene expressed in gastrointestinal epithelium. Proc Natl Acad Sci USA 93: 12445-12450, 1996.

10. Bakker AB, Phillips JH, Figdor CG and Lanier LL: Killer cell inhibitory receptors for MHC class I molecules regulate lysis of melanoma cells mediated by NK cells, gamma delta T cells, and antigen-specific CTL. J Immunol 160: 5239-5245, 1998.

11. Chang CC and Ferrone S: NK cell activating ligands on human malignant cells: Molecular and functional defects and potential clinical relevance. Semin Cancer Biol 16: 383-392, 2006.

12. Hilpert J, Grosse-Hovest L, Grunebach F, Buechele C, Nuebling T, Raum T, Steinle A and Salih HR: Comprehensive analysis of NKG2D ligand expression and release in leukemia: Implications for NKG2D-mediated NK cell responses. J Immunol 189: 1360-1371, 2012.

13. Bae JH, Kim JY, Kim MJ, Chang SH, Park YS, Son CH, Park SJ, Chung JS, Lee EY, Kim SH and Kang CD: Quercetin enhances susceptibility to NK cell-mediated lysis of tumor cells through induction of NKG2D ligands and suppression of HSP70. J Immunother 33: 391-401, 2010

14. Boissel N, Rea D, Tieng V, Dulphy N, Brun M, Cayuela JM, Rousselot P, Tamouza R, Le Bouteiller P, Mahon FX, et al: $\mathrm{BCR} / \mathrm{ABL}$ oncogene directly controls MHC class I chain-related molecule A expression in chronic myelogenous leukemia. J Immunol 176: 5108-5116, 2006.

15. Heo W, Lee YS and Bae JH: Inhibition of oncogenes affects the expression of NKG2D ligands in cancer cells. J Life Sci 23: 1216-1222, 2013.

16. Peinado C, Kang X, Hardamon C, Arora S, Mah S, Zhang H, Ngolab J and Bui JD: The nuclear factor- $\kappa$ B pathway down-regulates expression of the NKG2D ligand H60a in vitro: Implications for use of nuclear factor- $\kappa \mathrm{B}$ inhibitors in cancer therapy. Immunology 139: 265-274, 2013.

17. Park SW, Bae JH, Kim SD, Son YO, Kim JY, Park HJ, Lee CH, Park DY, Kim JY, Lee MK, et al: Comparison of level of NKG2D ligands between normal and tumor tissue using multiplex RT-PCR. Cancer Invest 25: 299-307, 2007.

18. Huang MJ, Cheng YC, Liu CR, Lin S and Liu HE: A small-molecule c-Myc inhibitor, 10058-F4, induces cell-cycle arrest, apoptosis, and myeloid differentiation of human acute myeloid leukemia. Exp Hematol 34: 1480-1489, 2006. 
19. Albajar M, Gómez-Casares MT, Llorca J, Mauleon I, Vaqué JP, Acosta JC, Bermudez A, Donato N, Delgado MD and León J: MYC in chronic myeloid leukemia: Induction of aberrant DNA synthesis and association with poor response to imatinib. Mol Cancer Res 9: 564-576, 2011.

20. Coulis CM, Lee C, Nardone V and Prokipcak RD: Inhibition of c-myc expression in cells by targeting an RNA-protein interaction using antisense oligonucleotides. Mol Pharmacol 57: 485-494, 2000.

21. Lozzio CB and Lozzio BB: Human chronic myelogenous leukemia cell-line with positive Philadelphia chromosome. Blood 45: 321-334, 1975.

22. Law JC, Ritke MK, Yalowich JC, Leder GH and Ferrell RE: Mutational inactivation of the p53 gene in the human erythroid leukemic K562 cell line. Leuk Res 17: 1045-1050, 1993.

23. Zindy F, Eischen CM, Randle DH, Kamijo T, Cleveland JL, Sherr CJ and Roussel MF: Myc signaling via the ARF tumor suppressor regulates p53-dependent apoptosis and immortalization. Genes Dev 12: 2424-2433, 1998.

24. Campoli M and Ferrone S: Tumor escape mechanisms: Potential role of soluble HLA antigens and NK cells activating ligands. Tissue Antigens 72: 321-334, 2008.

25. Dunn GP, Bruce AT, Ikeda H, Old LJ and Schreiber RD: Cancer immunoediting: From immunosurveillance to tumor escape. Nat Immunol 3: 991-998, 2002.
26. Eisele G, Wischhusen J, Mittelbronn M, Meyermann R, Waldhauer I, Steinle A, Weller M and Friese MA: TGF-beta and metalloproteinases differentially suppress NKG2D ligand surface expression on malignant glioma cells. Brain 129: 2416-2425, 2006.

27. Groh V, Wu J, Yee C and Spies T: Tumour-derived soluble MIC ligands impair expression of NKG2D and T-cell activation. Nature 419: 734-738, 2002.

28. Xu L, Chen X, Shen M, Yang DR, Fang L, Weng G, Tsai Y, Keng PC, Chen Y and Lee SO: Inhibition of IL-6-JAK/Stat3 signaling in castration-resistant prostate cancer cells enhances the NK cell-mediated cytotoxicity via alteration of PD-L1/NKG2D ligand levels. Mol Oncol 12: 269-286, 2018.

29. Gasser S, Orsulic S, Brown EJ and Raulet DH: The DNA damage pathway regulates innate immune system ligands of the NKG2D receptor. Nature 436: 1186-1190, 2005.

30. Nanbakhsh A, Pochon C, Mallavialle A, Amsellem S, Bourhis JH and Chouaib S: c-Myc regulates expression of NKG2D ligands ULBP $1 / 2 / 3$ in AML and modulates their susceptibility to NK-mediated lysis. Blood 123: 3585-3595, 2014.

This work is licensed under a Creative Commons Attribution-NonCommercial-NoDerivatives 4.0 International (CC BY-NC-ND 4.0) License. 